\title{
Date Time of Angina Pectoris Prompting Medical Attention
}

National Cancer Institute

\section{Source}

National Cancer Institute. Date Time of Angina Pectoris Prompting Medical Attention. NCI Thesaurus. Code C91344.

The date and time of the ang ina pectoris event requiring an individual to seek medical attention. 